\title{
A tolerability and safety analysis of adding granulocyte- macrophage colony-stimulating factor to local radiotherapy in a case series of seven patients with thoracic cancer
}

\author{
Bi-Cheng Wang ${ }^{1 \#}$, Peng-Cheng Li $^{1 \#}$, Bo-Hua Kuang ${ }^{1,2 \#}$, Zhan-Jie Zhang ${ }^{1 \#}$, Bo-Ya Xiao ${ }^{3,4}$, Guo-He Lin ${ }^{5}$, \\ Quentin Liu ${ }^{2}$
}

${ }^{1}$ Cancer Center, Union Hospital, Tongji Medical College, Huazhong University of Science and Technology, Wuhan, China; ${ }^{2}$ State Key Laboratory of Oncology in South China, Department of Experimental Research, Sun Yat-sen University Cancer Center, Collaborative Innovation Center for Cancer Medicine, Guangzhou, China; ${ }^{3}$ Eastern Hepatobiliary Surgery Hospital, Second Military Medical University, Shanghai, China; ${ }^{4}$ Department of Medical Psychology, Faculty of Psychology, Naval Medical University (Second Military Medical University), Shanghai, China; ${ }^{5}$ Department of Oncology, the Second Affiliated Hospital of Anhui Medical University, Hefei, China

Contributions: (I) Conception and design: BC Wang; (II) Administrative support: BC Wang, PC Li; (III) Provision of study materials or patients: BC Wang, PC Li; (IV) Collection and assembly of data: BC Wang, BY Xiao, GH Lin; (V) Data analysis and interpretation: BC Wang, BH Kuang, ZJ Zhang; (VI) Manuscript writing: All authors; (VII) Final approval of manuscript: All authors.

\#These authors contributed equally to this work.

Correspondence to: Bi-Cheng Wang, MD. Cancer Center, Union Hospital, Tongji Medical College, Huazhong University of Science and Technology, 1277 Jiefang Avenue, Wuhan 430022, China. Email: bcsnowell@163.com.

Background: Granulocyte-macrophage colony-stimulating factor (GM-CSF) has been demonstrated to improve the anti-cancer effects in combination with radiotherapy. However, the tolerability and safety of adding GM-CSF to radiotherapy in thoracic cancer patients need to be further explored.

Methods: Between June 2020 and Sep 2020, seven patients with thoracic cancer were treated with concurrent radiotherapy and GM-CSF (200 $\mu \mathrm{g}$ subcutaneously injected q.o.d during the radiotherapy). The primary endpoint was adverse event.

Results: Of seven enrolled patients, four were non-small cell lung cancer, two were small cell lung cancer, and the other one patient was thymic carcinoma. The total dose of GM-CSF that each patient received was at least $3000 \mu \mathrm{g}$. All patients had finished the radiotherapy and GM-CSF injection and suffered one or more any grade adverse events. Only one patient had a grade $\geq 3$ hematological adverse event (lymphocytopenia). Grade $\geq 3$ non-hematological toxicities were not observed during the combination treatment. The highest cell counts of white blood cell, neutrophile granulocyte, and monocyte across the treatment were $22.38 \times 10^{9} / \mathrm{L}$, $18.65 \times 10^{9} / \mathrm{L}$, and $1.28 \times 10^{9} / \mathrm{L}$ respectively.

Conclusions: The combination therapy of radiotherapy and GM-CSF (200 $\mu \mathrm{g}$ subcutaneously q.o.d) is tolerable and safe. Further studies are warranted to confirm the effects and optimal total GM-CSF injection doses in the combination of radiotherapy in thoracic cancer patients.

Keywords: Granulocyte-macrophage colony-stimulating factor (GM-CSF); tolerability; safety; thoracic cancer; case series

Submitted Nov 11, 2020. Accepted for publication Feb 24, 2021.

doi: 10.21037/apm-20-2248

View this article at: http://dx.doi.org/10.21037/apm-20-2248

\footnotetext{
^ ORCID: 0000-0002-4598-7721.
} 


\section{Introduction}

In the treatment of early-stage cancer, radiotherapy is a highly efficacious strategy. For patients with stage I non-small cell lung cancer (NSCLC), stereotactic body radiotherapy (SBRT) has shown comparable survival outcomes versus surgery (1). However, there is another voice demonstrating that the overall survival (OS) and disease-free survival (DFS) for patients with stage I NSCLC in the surgery group were slightly superior to those in the SBRT group (2). Therefore, radiotherapy alone might not be enough for patients with thoracic cancer, especially for those with advanced disease.

In order to decrease the incidences of distant metastasis and prolong progression-free survival (PFS), systemic chemotherapy is administrated. Immune checkpoint inhibitors (ICIs) have revolutionized the treatment of cancer patients during the recent ten years. PD-1 inhibitors, pembrolizumab and nivolumab, have been recommended to NSCLC. Moreover, PD-L1 inhibitors, atezolizumab and durvalumab, are preferred for small cell lung cancer (SCLC). Additionally, anti-PD-1/PD-L1 therapy is also a promising treatment option for patients with thymic carcinoma $(3,4)$.

Currently, numerous clinical studies are conducted to explore the combination strategy of radiotherapy and immunotherapy. Clinicians and researchers considered that adding ICIs to radiotherapy and/or chemotherapy might be a more efficacious strategy to increase the chance of surgery for locoregionally advanced thoracic cancer patients $(5,6)$. Combined with radiotherapy, immunotherapy could provide a better immunological efficacy called abscopal effect, in which CD4 and CD8 positive T cells exert critical roles $(7,8)$. Granulocyte-macrophage colonystimulating factor (GM-CSF), the key factor, is a cytokine that promotes maturation of dendritic cells for antigen presentation and generates $\mathrm{T}$ - and B-lymphocyte anticancer response. Previous studies indicated that GM-CSF with radiotherapy product abscopal effect in metastatic cancer (9). On the other hand, combining ipilimumab with GM-CSF significantly improves the survival of metastatic melanoma (10).

In published studies, the common application of GMCSF was $125 \mu \mathrm{g} / \mathrm{m}^{2}$ or $250 \mu \mathrm{g}$ subcutaneously injected for consecutive 14 days and no severe adverse event was reported $(9,10)$. However, in a meeting report (11), Liu found that eight of 24 enrolled patients suffered high fever in the combination therapy of radiotherapy and GM-CSF $\left(125 \mu \mathrm{g} / \mathrm{m}^{2}\right.$ from $\mathrm{d} 8$ to $\left.\mathrm{d} 22\right)$. In the real world, when patients experience high fever, whether the injection of GM-CSF is continuously executed remains contradictory.

Therefore, in this study, we intend to exhibit a novel and tolerable GM-CSF therapeutic regimen in patients with thoracic cancer. We present the following article in accordance with the AME Case Series reporting checklist (available at http://dx.doi.org/10.21037/apm-20-2248).

\section{Methods}

\section{Patients}

A total of seven patients who were pathologically diagnosed with thoracic cancer at Union Hospital, Tongji Medical College, Huazhong University of Science and Technology between June 2020 and September 2020 were retrospectively enrolled in the present study. Four patients were histologically diagnosed with NSCLC, two were SCLC, and the other one was thymic carcinoma. The study was conducted in accordance with the Declaration of Helsinki (as revised in 2013). The study design was approved by the Ethics Committee of the Union Hospital, Tongji Medical College, Huazhong University of Science and Technology (No. [2020]IEC-J071). All patients had written informed consent to participate in this study.

\section{Study design and treatment strategy}

The Eastern Cooperative Oncology Group (ECOG) performance status of eligible patients should be 0 or 1 and life expectancy was expected more than three months. Patients received single radiotherapy (times $\geq 25$, lung V $20<30 \%$ ) and were administered $200 \mu \mathrm{g}$ GM-CSF (Te er li, Xiamen Amoytop Biotech) subcutaneous injection q.o.d from the initiation of radiotherapy, followed by every Monday, Wednesday, and Friday GM-CSF injection till the end of radiotherapy (Figure 1). GM-CSF was discontinued for documented white blood cell count greater than $30 \times 10^{9} / \mathrm{L}$ and for any significant toxicity to be caused by GM-CSF, like fever over 39.0 degrees $\mathrm{C}$ or 102.3 degrees F). Patients who received concomitant radiotherapy with chemotherapy or targeting therapy were excluded.

\section{Outcomes}

The primary endpoint was adverse event. Safety was assessed by an evaluation of the incidence of hematological (anemia, leukocytosis, lymphopenia, thrombocytopenia, 


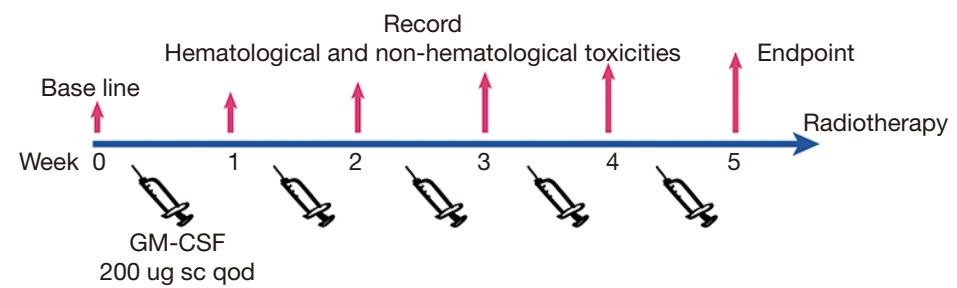

Figure 1 Timeline events of enrolled thoracic cancer patients.

Table 1 Characteristics of the included patients in the study

\begin{tabular}{lc}
\hline Characteristic & No. (\%) \\
\hline Duration & Jun 2020-Sep 2020 \\
ECOG performance status & $7[100]$ \\
0 & $0(0)$ \\
1 & $68[58-79]$ \\
Age, median (range), y & $6(85.7)$ \\
Sex & $1(14.3)$ \\
Men & \\
Women & \\
Cancer types & $4(57.1)$ \\
Non-small cell lung cancer & $2(28.6)$ \\
Small cell lung cancer & $1(14.3)$ \\
Thymic carcinoma & $7[100]$ \\
Total GM-CSF $\geq 3,000 ~ \mu \mathrm{g}$ & $2(28.6)$ \\
Proposed discontinuing &
\end{tabular}

ECOG, Eastern Cooperative Oncology Group; GM-CSF, granulocyte-macrophage colony-stimulating factor.

leucopenia, neutropenia, increased cTnI, increased creatinine, increased alanine aminotransferase/aspartate aminotransferase levels, etc.) and non-hematological (hypertension, esophagitis, fatigue, nausea, vomiting, myalgia, cough, etc.) toxicities, which were graded according to the Common Terminology Criteria for adverse events (CTCAE, version 5.0). Since CD4 and CD8 positive $\mathrm{T}$ cells might indirectly reflect the treatment responses, these two cohorts of lymphocytes were tested before and at the end of the radiotherapy. Computed tomography (CT) was used to evaluate the toxicities or tumor response if patients experience fever.

\section{Statistical analysis}

Comparisons for CD4+ T cells and CD8+ T cells between post-treatment and baseline were calculated by GraphPad Prism 6 software by paired two-tailed Student's $t$-test. A P value $<0.05$ was considered statistically significant.

\section{Results}

\section{Characteristics and tolerability}

From Jun 2020 to Sep 2020, of seven thoracic cancer patients treated with combination therapy of radiotherapy and GM-CSF, six were men and one was a woman. All patients had an ECOG performance status of 0 . The median age was 68 years old (range, 58-79). Three types of cancer were enrolled, including NSCLC $(n=4)$, SCLC $(n=2)$, and thymic carcinoma $(n=1)$. The total GM-CSF dose of each patient was over 3,000 $\mu \mathrm{g}$. Among the seven patients, two had proposed to discontinue the injection of GM-CSF because they were not sure about the benefits of GM-CSF. However, they finished the combination therapy finally. Table 1 displays the basic characteristics of these patients.

\section{Hematological toxicities}

Only one patient with grade 3 lymphopenia was detected. The most frequently reported hematological toxicities of any grade were lymphopenia $(n=6)$ and thrombocytopenia $(\mathrm{n}=3)$. Leucopenia (grade $2, \mathrm{n}=1$ ), neutropenia (grade 2, $\mathrm{n}=1$ ), increased cTnI (grade $1, \mathrm{n}=1$ ), and increased creatinine (grade $1, \mathrm{n}=1$ ) were observed. We did not find anemia, leukocytosis (grade $\geq 3$ ), or increased alanine aminotransferase/aspartate aminotransferase levels.

Figure $2 A$ showed that the median counts of white blood cell, neutrophile granulocyte, and monocyte were $12.51 \times 10^{9} / \mathrm{L}$ (range, $12.17-22.38 \times 10^{9} / \mathrm{L}$ ), $11.20 \times 10^{9} / \mathrm{L}$ 

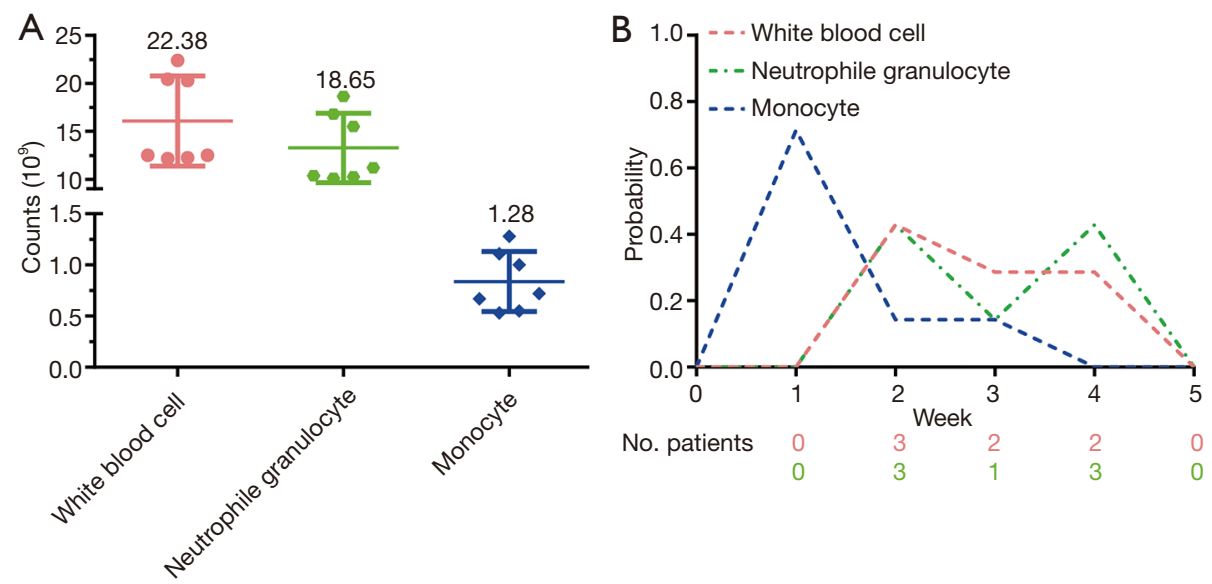

Figure 2 The variation characteristics of white blood cell, neutrophile granulocyte, and monocyte. (A) Highest values of white blood cell, neutrophile granulocyte, and monocyte during the treatment; (B) the percentages of patients achieving the highest values of white blood cell, neutrophile granulocyte, and monocyte at each week.
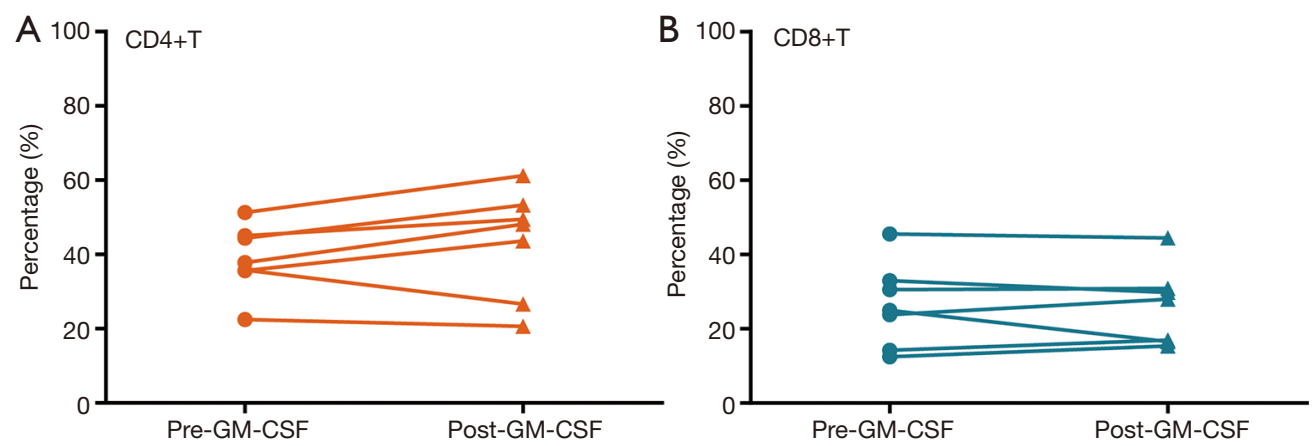

Figure 3 Radiotherapy plus GM-CSF had no significant effect on the percentages of CD4 and CD8 T cells. GM-CSF, granulocytemacrophage colony-stimulating factor.

(range, $10.10-18.65 \times 10^{9} / \mathrm{L}$ ), and $0.72 \times 10^{9} / \mathrm{L}$ (range, $\left.0.53-1.28 \times 10^{9} / \mathrm{L}\right)$ respectively. At week 1 , five of the seven patients reached their highest levels of monotherapy. Three patients achieved the highest levels of white blood cell at week 2. Three patients each reached the highest levels of neutrophile granulocyte at week 2 and 4 (Figure $2 B$ ).

\section{Non-bematological toxicities}

No grade 3 or more non-hematological toxicities were showed during the combination therapy. The most frequently reported grade 1-2 non-hematological toxicities were hypertension $(n=5)$ and esophagitis $(n=3)$, followed by fatigue $(n=2)$, nausea/vomiting $(n=2)$, myalgia $(n=2)$, and cough $(n=2)$. One patient had experienced grade 1 fever. Anaphylaxis, injection site reaction, arthralgia, mucositis, and autoimmune disorder were not recorded.

\section{Percentage of T lymphocytes}

Compared to the baseline values, the percentage of $\mathrm{CD} 4$ $(\mathrm{P}=0.166)$ and $\mathrm{CD} 8(\mathrm{P}=0.842) \mathrm{T}$ cells showed no significant difference after the combination therapy of radiotherapy and GM-CSF (Figure 3).

\section{Discussion}

In this study, we showed that radiotherapy plus GMCSF $200 \mu$ g subcutaneously q.o.d is well tolerable with a safety profile. Lymphopenia and hypertension are the most common hematological and non-hematological adverse events respectively. 
Table 2 Hematological toxicities during the combination therapy of radiotherapy and GM-CSF

\begin{tabular}{lcccc}
\hline \multirow{2}{*}{$\begin{array}{l}\text { Hematological } \\
\text { toxicities }\end{array}$} & \multicolumn{4}{c}{ No. patients } \\
\cline { 2 - 5 } Lymphopenia & 1 & 4 & 1 & 6 \\
Thrombocytopenia & 1 & 2 & 0 & 3 \\
Leucopenia & 0 & 1 & 0 & 1 \\
Neutropenia & 0 & 1 & 0 & 1 \\
cTnl increased & 1 & 0 & 0 & 1 \\
Cr increased & 1 & 0 & 0 & 1 \\
Anemia & 0 & 0 & 0 & 0 \\
Leukocytosis & - & - & 0 & 0 \\
ALT/AST increased & 0 & 0 & 0 & 0 \\
\hline
\end{tabular}

cTnI, cardiac troponin I; Cr, creatinine; ALT, alanine aminotransferase; AST, aspartate aminotransferase.

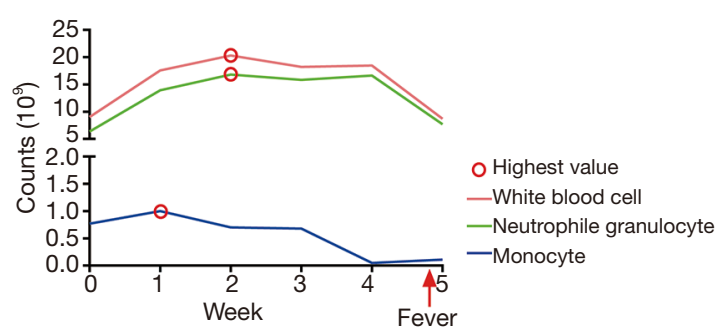

Figure 4 Cell counts curves of white blood cell, neutrophile granulocyte, and monocyte in the non-small cell lung cancer patient.

Key elements in the immune response activated by radiotherapy include immunostimulatory cytokines, dendritic cells maturation, and $\mathrm{T}$ lymphocyte recruitment and stimulation (12). CD8 $\mathrm{T}$ cells have a critical role in mediating anti-tumor effects. The infiltration of CD8 T cells in the tumor microenvironment is associated with a better prognosis in multiple types of cancer $(13,14)$. Furthermore, CD4 $\mathrm{T}$ cells also play a central role in tumor control and correlate with good clinical prognosis. Although GM-CSF is an important factor for dendritic cell development under anti-tumor conditions, the application of GM-CSF failed to against the lymphopenia caused by radiotherapy in our study (Table 2). Fortunately, no statistical differences were observed after the combination therapy in terms of the percentage of CD4 and CD8 positive $\mathrm{T}$ cells.
Since the lymphocyte count decreased, the efficacy of adding immunotherapy to radiotherapy remains controversial. In Golden's study, abscopal effects were observed when patients received localized radiotherapy and GM-CSF. But the enrolled patients were given systemic chemotherapy as well (9). Therefore, it might be an unprecise conclusion that single radiotherapy plus GM-CSF produced abscopal responses. A recent phase 2 clinical trial conducted by McBride showed no improvement in response and no evidence of abscopal effects with the combination of anti-PD-1 therapy and stereotactic body radiotherapy versus anti-PD-1 therapy alone in advanced head and neck squamous cell carcinoma (15). In another randomized clinical trial, researchers found that anti-CTLA-4 therapy plus GM-CSF significantly improved survival in metastatic melanoma patients compared to anti-CTLA-4 therapy (10).

Therefore, we suspect whether the triple combination therapy of radiotherapy, ICI inhibitors, and GM-CSF could have higher clinical effects. In the case study reported by Zhao et al., a patient with refractory metastatic esophageal squamous cell carcinoma received stereotactic body radiotherapy plus anti-PD-1 therapy (sintilimab) and GM-CSF. Although abscopal effects were observed, the patient died of severe pneumonia (16). In this case report, GM-CSF $200 \mu \mathrm{g}$ daily was subcutaneously injected for 14 days. However, the authors did not exhibit the incidence of leukocytosis and the cell counts of white blood cell, neutrophile granulocyte, and monocyte.

GM-CSF is commonly used to prevent hospitalization for febrile neutropenia during systemic chemotherapy or local radiotherapy $(17,18)$. Additionally, fever is one of the frequent adverse events after GM-CSF injection that we mainly focus on, because it might be associated with the high level of white blood cell and autoimmune disorders. We are eager to know the highest number of white blood cell, neutrophile granulocyte, and monocyte during the GM-CSF treatment. In our study, the highest cell counts were $22.38 \times 10^{9} / \mathrm{L}$ for white blood cell, $18.65 \times 10^{9} / \mathrm{L}$ for neutrophile granulocyte, and $1.28 \times 10^{9} / \mathrm{L}$ for monocyte, with good tolerability (Figure $2 A$ ). Considering the high incidence of fever in the combination therapy of radiotherapy and GM-CSF (11), we suggest that the reasonability of consecutive 14 days injection of GM-CSF is needed to be challenged.

Only one patient in our study occurred fever. The details of white blood cell, neutrophile granulocyte, and monocyte are shown in Figure 4. After a week of combination therapy, the monocyte reached the highest value. Then the white 

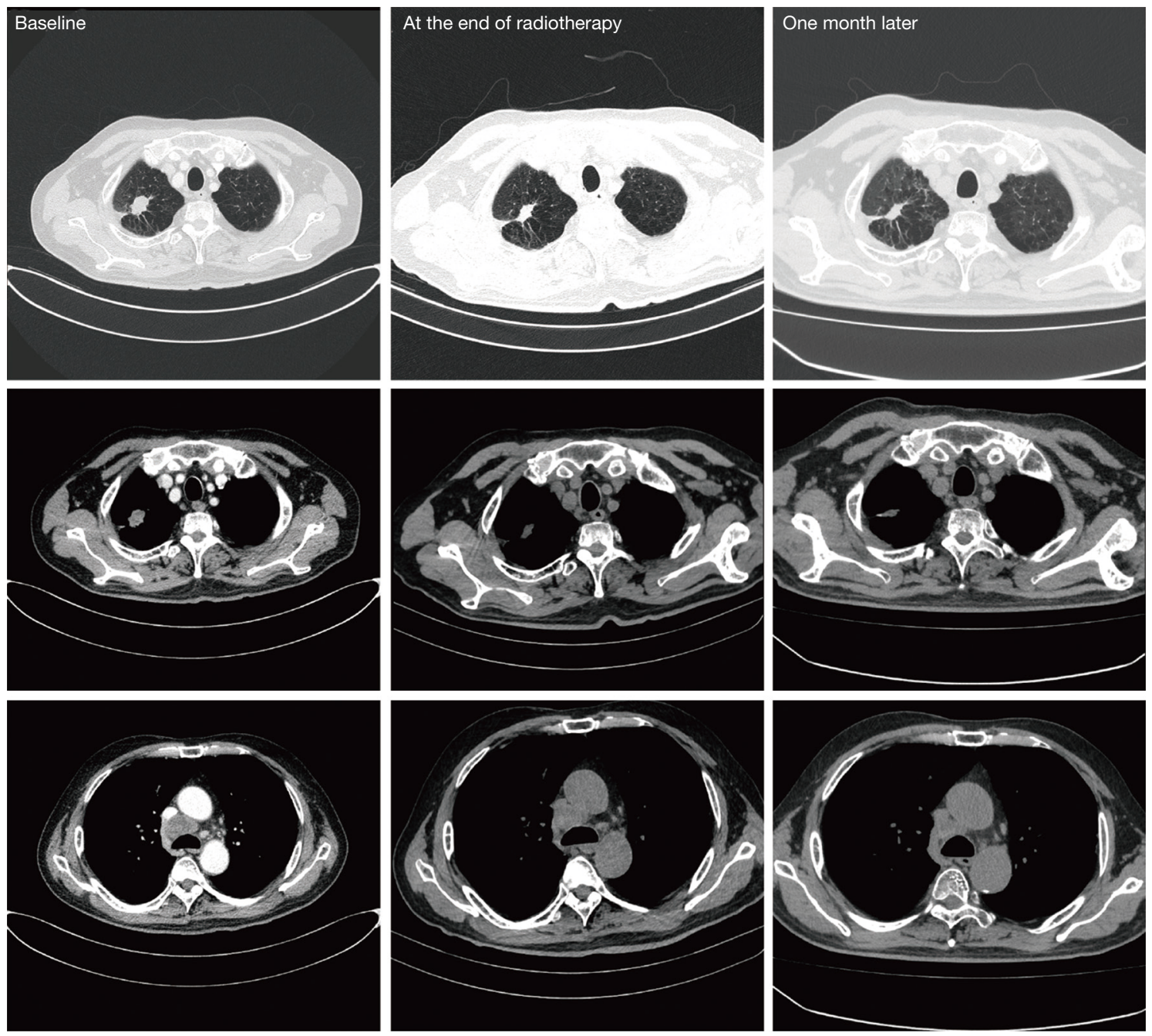

Figure 5 The non-small cell lung cancer patient who suffered fever with a partial response but without pneumonia.

blood cell and neutrophile granulocyte reached the highest value at week 2 . Nonetheless, the transient grade 1 fever $\left(38.2{ }^{\circ} \mathrm{C}\right)$ happened at the end of radiotherapy. Thus, we did not deem the fever was directly associated with radiotherapy or GM-CSF. The chest CT scan did not find pneumonia either (Figure 5).

There were several limitations in this study. First, this was a retrospective single-arm study, thus selection bias of patients should be existed. Second, considering that the addition of single GM-CSF treatment to radiotherapy might not strongly elevated the efficacy of radiotherapy and did not show significant improvement on the percentage of CD 4 and CD 8 positive T cells, anti-PD-1/PD-L1 therapy is recommended to the future clinical studies. Third, the assessment of treatment responses was absent in this study owing to the different therapeutic purposes of radiotherapy. However, based on our study, GM-CSF $200 \mu \mathrm{g}$ subcutaneously q.o.d could be an alternative treatment strategy to ensure the tolerability and continuity of GM-CSF therapy.

In summary, radiotherapy combined with GM-CSF $200 \mu \mathrm{g}$ subcutaneously q.o.d is tolerable and feasible. Future clinical studies are warranted to explore the benefits and safety profiles of adding ICIs to the combination therapy of radiotherapy and GM-CSF.

\section{Acknowledgments}

We thank the SNOWELL studio for helping to provide statistical support and improve the language. 
Funding: This study was supported by the Hubei Provincial Natural Science Foundation (Grant number: 2020CFB397 to Bi-Cheng Wang) and the Independent Innovation Foundation of Wuhan Union Hospital (Grant number: 2019-109 to Bi-Cheng Wang).

\section{Footnote}

Reporting Checklist: The authors have completed the AME Case Series reporting checklist. Available at http://dx.doi. org/10.21037/apm-20-2248

Data Sharing Statement: Available at http://dx.doi. org/10.21037/apm-20-2248

Conflicts of Interest: All authors have completed the ICMJE uniform disclosure form (available at http://dx.doi. org/10.21037/apm-20-2248). BCW reports grants from Hubei Provincial Natural Science Foundation, grants from Independent Innovation Foundation of Wuhan Union Hospital, outside the submitted work. The other authors have no conflicts of interest to declare.

Ethical Statement: The authors are accountable for all aspects of the work in ensuring that questions related to the accuracy or integrity of any part of the work are appropriately investigated and resolved. The study was conducted in accordance with the Declaration of Helsinki (as revised in 2013). The study design was approved by the Ethics Committee of the Union Hospital, Tongji Medical College, Huazhong University of Science and Technology (No. [2020]IEC-J071). All patients had written informed consent to participate in this study.

Open Access Statement: This is an Open Access article distributed in accordance with the Creative Commons Attribution-NonCommercial-NoDerivs 4.0 International License (CC BY-NC-ND 4.0), which permits the noncommercial replication and distribution of the article with the strict proviso that no changes or edits are made and the original work is properly cited (including links to both the formal publication through the relevant DOI and the license). See: https://creativecommons.org/licenses/by-nc-nd/4.0/.

\section{References}

1. Mayne NR, Lin BK, Darling AJ, et al. Stereotactic Body Radiotherapy Versus Delayed Surgery for Early-stage
Non-small-cell Lung Cancer. Ann Surg 2020;272:925-29.

2. Tomita N, Okuda K, Osaga S, et al. Surgery versus stereotactic body radiotherapy for clinical stage I nonsmall-cell lung cancer: propensity score-matching analysis including the ratio of ground glass nodules. Clin Transl Oncol 2021;23:638-47.

3. Giaccone G, Kim C, Thompson J, et al. Pembrolizumab in patients with thymic carcinoma: a single-arm, singlecentre, phase 2 study. Lancet Oncol 2018;19:347-55.

4. Rajan A, Heery CR, Thomas A, et al. Efficacy and tolerability of anti-programmed death-ligand 1 (PD-L1) antibody (Avelumab) treatment in advanced thymoma. J Immunother Cancer 2019;7:269.

5. Dickhoff C, Senan S, Schneiders FL, et al. Ipilimumab plus nivolumab and chemoradiotherapy followed by surgery in patients with resectable and borderline resectable T34N0-1 non-small cell lung cancer: the INCREASE trial. BMC Cancer 2020;20:764.

6. Hida T, Yamaguchi T. Advances in immunotherapy for stage III non-small cell lung cancer: moving immune checkpoint inhibitors to the front lines concurrently with chemoradiotherapy? J Thorac Dis 2020;12:4549-52.

7. Lin $W, X u Y$, Chen $X$, et al. Radiation-induced small extracellular vesicles as "carriages" promote tumor antigen release and trigger antitumor immunity. Theranostics 2020;10:4871-84.

8. Min Y, Roche KC, Tian S, et al. Antigen-capturing nanoparticles improve the abscopal effect and cancer immunotherapy. Nat Nanotechnol 2017;12:877-82.

9. Golden EB, Chhabra A, Chachoua A, et al. Local radiotherapy and granulocyte-macrophage colonystimulating factor to generate abscopal responses in patients with metastatic solid tumours: a proof-of-principle trial. Lancet Oncol 2015;16:795-803.

10. Hodi FS, Lee S, McDermott DF, et al. Ipilimumab plus sargramostim vs ipilimumab alone for treatment of metastatic melanoma: a randomized clinical trial. JAMA 2014;312:1744-53.

11. Liu M, Cai X, Zeng Y. The Abscopal Effects of the Combination of Radiotherapy and GM-CSF for Patients with Metastatic Thoracic Cancers. J Thorac Oncol 2019;14:S980.

12. Barker HE, Paget JT, Khan AA, et al. The tumour microenvironment after radiotherapy: mechanisms of resistance and recurrence. Nat Rev Cancer 2015;15:409-25.

13. Fridman WH, Pages F, Sautes-Fridman C, et al. The immune contexture in human tumours: impact on clinical 
outcome. Nat Rev Cancer 2012;12:298-306.

14. Reiser J, Banerjee A. Effector, Memory, and Dysfunctional CD8(+) T Cell Fates in the Antitumor Immune Response. J Immunol Res 2016;2016:8941260.

15. McBride S, Sherman E, Tsai CJ, et al. Randomized Phase II Trial of Nivolumab With Stereotactic Body Radiotherapy Versus Nivolumab Alone in Metastatic Head and Neck Squamous Cell Carcinoma. J Clin Oncol 2021;39:30-7.

16. Zhao X, Kong Y, Zhang L. Anti-PD-1 Immunotherapy Combined With Stereotactic Body Radiation Therapy and GM-CSF as Salvage Therapy in a PD-L1-Negative Patient With Refractory Metastatic Esophageal Squamous
Cell Carcinoma: A Case Report and Literature Review. Front Oncol 2020;10:1625.

17. Heaney ML, Toy EL, Vekeman F, et al. Comparison of hospitalization risk and associated costs among patients receiving sargramostim, filgrastim, and pegfilgrastim for chemotherapy-induced neutropenia. Cancer 2009; 115:4839-48.

18. Weycker D, Malin J, Barron R, et al. Comparative effectiveness of filgrastim, pegfilgrastim, and sargramostim as prophylaxis against hospitalization for neutropenic complications in patients with cancer receiving chemotherapy. Am J Clin Oncol 2012;35:267-74.

Cite this article as: Wang $\mathrm{BC}$, Li PC, Kuang BH, Zhang ZJ, Xiao BY, Lin GH, Liu Q. A tolerability and safety analysis of adding granulocyte-macrophage colony-stimulating factor to local radiotherapy in a case series of seven patients with thoracic cancer. Ann Palliat Med 2021;10(4):4193-4200. doi: 10.21037/apm20-2248 